•综述・

\title{
陆生食肉动物竞争与共存研究概述
}

\author{
李治霖@1,2，多立安 ${ }^{1,2}$ ，李戟@ $3^{*}$ ，王天明@4,5,6*
}

1. 天津师范大学生命科学学院天津市动植物抗性重点实验室, 天津 300387; 2. 天津师范大学生命科学学院天津市动物多样性保护与利用 重点实验室, 天津 300387; 3. 北京大学生命科学学院, 北京 $100871 ; 4$. 生物多样性与生态工程教育部重点实验室, 北京 100875; 5. 东北虎 豹国家公园保护生态学国家林草局重点实验室, 北京 100875 ; 6. 北京师范大学生命科学学院, 北京 100875

摘要: 陆生食肉动物(食肉目哺乳动物, 以下简称食肉动物)作为食物链与营养级的高位物种对维持生态系统结构与功能稳 定性起到重要作用。过度人类干扰已在全球范围内造成食肉动物种群数量剧烈下降和栖息地质量显著退化, 探究食肉动物 的区域共存机制对理解生物群落构建、濒危物种保护与管理具有重要意义。本文通过梳理100余篇有关食肉动物在空间、时 间和营养3个生态位维度上相互作用的研究，分析了体型大小、猎物组成、种群结构、环境差异、人类干扰和气候变化等因 素对食肉动物种间关系和区域共存的影响, 并对今后食肉动物区域共存研究中亟需解决的问题进行了展望。食肉动物通过 生态位分离达到共存并没有单一的理论解释，猎物、栖息地和人类干扰等因素可以调节食肉动物相互作用关系并直接或间 接地影响共存, 共同适应在食肉动物区域共存中具重要作用。食肉动物区域共存是经过长期演化形成的相对稳定状态, 需要 以动态的眼光去审视。要明晰生态位重叠与区域共存机制的区别与联系, 在理解生态位分离的基础上, 结合生活史、家域和 行为等对食肉动物共存进行综合分析。

关键词：食肉动物; 生态位分离; 物种共存; 集团内捕食理论; 种间关系

李治霖, 多立安, 李晟, 王天明 (2021) 陆生食肉动物竞争与共存研究概述. 生物多样性, 29, 81-97. doi: 10.17520/biods.2020359.

Li ZL, Duo LA, Li S, Wang TM (2021) Competition and coexistence among terrestrial mammalian carnivores. Biodiversity Science, 29, 81-97. doi: 10.17520/biods.2020359.

\section{Competition and coexistence among terrestrial mammalian carnivores}

\author{
Zhilin $\mathrm{Li}^{\text {(D) } 1,2}$, Li’an Duo ${ }^{1,2}$, Sheng $\mathrm{Li}^{\text {(D)3* }}{ }^{\text {, Tianming Wang }}{ }^{\text {(D) 4,5,6* }}$
}

1 Tianjin Key Laboratory of Animal and Plant Resistance, College of Life Sciences, Tianjin Normal University, Tianjin 300387

2 Tianjin Key Laboratory of Conservation and Utilization of Animal Diversity, College of Life Sciences, Tianjin Normal University, Tianjin 300387

3 School of Life Sciences, Peking University, Beijing 100871

4 Ministry of Education Key Laboratory for Biodiversity Science and Engineering, Beijing 100875

5 National Forestry and Grassland Administration Key Laboratory for Conservation Ecology in the Northeast Tiger and Leopard National Park, Beijing 100875

6 College of Life Sciences, Beijing Normal University, Beijing 100875

\section{ABSTRACT}

Background \& Aim: Carnivores play an important role in maintaining the structural and functional stability of ecosystems. Increasing anthropogenic pressure has resulted in dramatic population decline and habitat degradation of carnivores worldwide. Exploring regional coexistence mechanisms of carnivore populations is important to understand how mammal communities assemble, along with protecting and managing endangered species. This review summarizes the interactions of terrestrial carnivores along three dimensions (i.e., spatial, temporal and dietary), of their ecological niches, analyzing the influence of factors such as body size, prey composition, environmental difference, human disturbance and climate change on the coexistence of carnivores based on more than 100 related studies.

Review Results: We put forward the problems to be addressed for current study of carnivore coexistence. This study demonstrates that there is no single theoretical explanation for carnivore coexistence through niche separation. Prey,

收稿日期: 2020-09-09; 接受日期: 2020-12-29

基金项目：国家自然科学基金(31971539)、科技部国家重点研发计划(2019FY101702)和天津师范大学校级科研项目(52XB2010)

*共同通讯作者 Co-authors for correspondence. E-mail: shengli@pku.edu.cn; wangtianming@bnu.edu.cn 
habitat and human disturbance may mediate the intensity of carnivore interactions and then influence their coexistence directly and indirectly. We also emphasize the critical role of co-adaptation for carnivore coexistence. Carnivore coexistence is a relatively stable state of long-term evolution, which needs to be examined dynamically.

Perspectives: We should correctly understand the differences and connections between niche overlap and coexistence. Besides niche separation, carnivores' life history, movement and behavior should also be considered to interpret their coexistence.

Key words: carnivores; niche segregation; species coexistence; intraguild predation theory; interspecific relationship

陆生食肉动物通常指食肉目哺乳动物(以下简 称食肉动物), 它们作为陆地生态系统的重要组成 部分, 在维持群落结构和物种多样性上具有重要作 用。一般地, 根据成体体型大小可把食肉动物分为 大型食肉动物(体重 $\geq 15 \mathrm{~kg}$ )与小型食肉动物 $(<15$ kg) (表1), 根据其在特定生态系统营养级结构中的 位置可分为优势性食肉动物(dominant carnivores)与 次要竞争者(subordinate competitors) (Ripple et al, 2014; Wallach et al, 2015)。目前全球共记录陆生食 肉目哺乳动物 13 科 250 种, 其中大型食肉动物 31 种, 小型食肉动物219种(Hunter, 2020)。

随着全球人类活动强度的不断加剧, 自然生境 遭到持续破坏与干扰, 致使大量食肉动物栖息地面 临破碎化和岛屿化的问题(Crooks et al, 2017; Wang et al, 2017)。近期有研究显示, 全球71\%的大型食肉 动物种群数量持续减少, $60 \%$ 的大型食肉动物现存 分布区不到其历史分布区面积的一半(Ripple et al, 2014)。过度的捕猎、放牧、森林采伐、城镇建设等 活动严重降低了食肉动物的生态适合度, 并潜在影 响了种间相互作用(Soh et al, 2014; Li et al, 2017; Miller \& Schmitz, 2019)。野生动物管理者在推动和 促进食肉动物群落及栖息地恢复的同时, 还需兼顾
当地居民的民生发展，这为食肉动物的管理与保护 提出了新的挑战(Wang et al, 2018; Farrington \& Tsering, 2019)。与此同时, 全球性气候变化导致的 自然栖息地改变也可能对食肉动物生境适宜性及 种间相互作用带来新的挑战(Lovari et al, 2013)。例 如, 气候变化使南亚山区的雪线上升, 改变了猎物 和栖息地范围, 进而增大了雪豹(Panthera uncia)与 豹(P. pardus)的相遇概率, 有可能加剧二者之间的 种间竞争(Lovari et al, 2013)。

在此背景下, 食肉动物怎样通过不同生态位维 度上的交互作用达到区域共存, 近年来成为保护生 物学及动物生态学的热点问题。Grinnell (1917)首先 使用生态位(niche)一词来描述动物在生态系统中的 位置。空间、时间和营养生态位是物种生态位构建 中典型的 3 个维度, 独立地描述了动物的生态位置 和资源使用。当多个物种共存于同一个群落时, 它 们在生态位的各个维度上就不可避免地发生相互 作用(Sæbø, 2016)。空间、时间和营养生态位为食肉 动物提供了 3 个可变的坐标维度, 动物可以在种间 竞争中通过适应或行为改变来调整其在各个维度 上的生态幅宽度与范围, 获取最大化收益(Schoener, 1974; Bruno et al, 2003), 并减少物种之间的竞争作

表1 依据体重的食肉动物分类及其种群调控方式与生态作用

Table 1 Carnivore classification based on body weight, corresponding with population regulation mode and their ecological function

\begin{tabular}{|c|c|c|c|c|}
\hline $\begin{array}{l}\text { 分类 } \\
\text { Classification }\end{array}$ & $\begin{array}{l}\text { 体重 } \\
\text { Body weight }\end{array}$ & $\begin{array}{l}\text { 种群调节方式 } \\
\text { Population regulation }\end{array}$ & $\begin{array}{l}\text { 生态作用 } \\
\text { Ecological function }\end{array}$ & $\begin{array}{l}\text { 参考文献 } \\
\text { References }\end{array}$ \\
\hline $\begin{array}{l}\text { 大型食肉动 } \\
\text { 物 } \\
\text { Large } \\
\text { carnivore }\end{array}$ & $\geq 15 \mathrm{~kg}$ & $\begin{array}{l}\text { 种群内部调节 } \\
\text { Intra-population regulation }\end{array}$ & $\begin{array}{l}\text { 控制草食类动物过度繁殖、维护植被结构、防止外来物种入侵 } \\
\text { 及疾病传播、降低碳排放 } \\
\text { Control over-reproduction of herbivores, maintain vegetation } \\
\text { structure, prevent exotic species invasion and diseases spread, and } \\
\text { reduce carbon emissions }\end{array}$ & Ripple et al, 2014 \\
\hline $\begin{array}{l}\text { 小型食肉动 } \\
\text { 物 } \\
\text { Small } \\
\text { carnivore }\end{array}$ & $<15 \mathrm{~kg}$ & $\begin{array}{l}\text { 外部环境调节 } \\
\text { Environmental } \\
\text { regulation }\end{array}$ & $\begin{array}{l}\text { 控制物质循环、塑造猎物群落构成、促进植被群落更新、调控 } \\
\text { 疾病传播 } \\
\text { Control material circulation, shape the composition of prey } \\
\text { communities, promote the regeneration of vegetation communities, } \\
\text { and regulate the diseases spread }\end{array}$ & $\begin{array}{l}\text { Salo et al, 2007; } \\
\text { Roemer et al, } 2009\end{array}$ \\
\hline
\end{tabular}




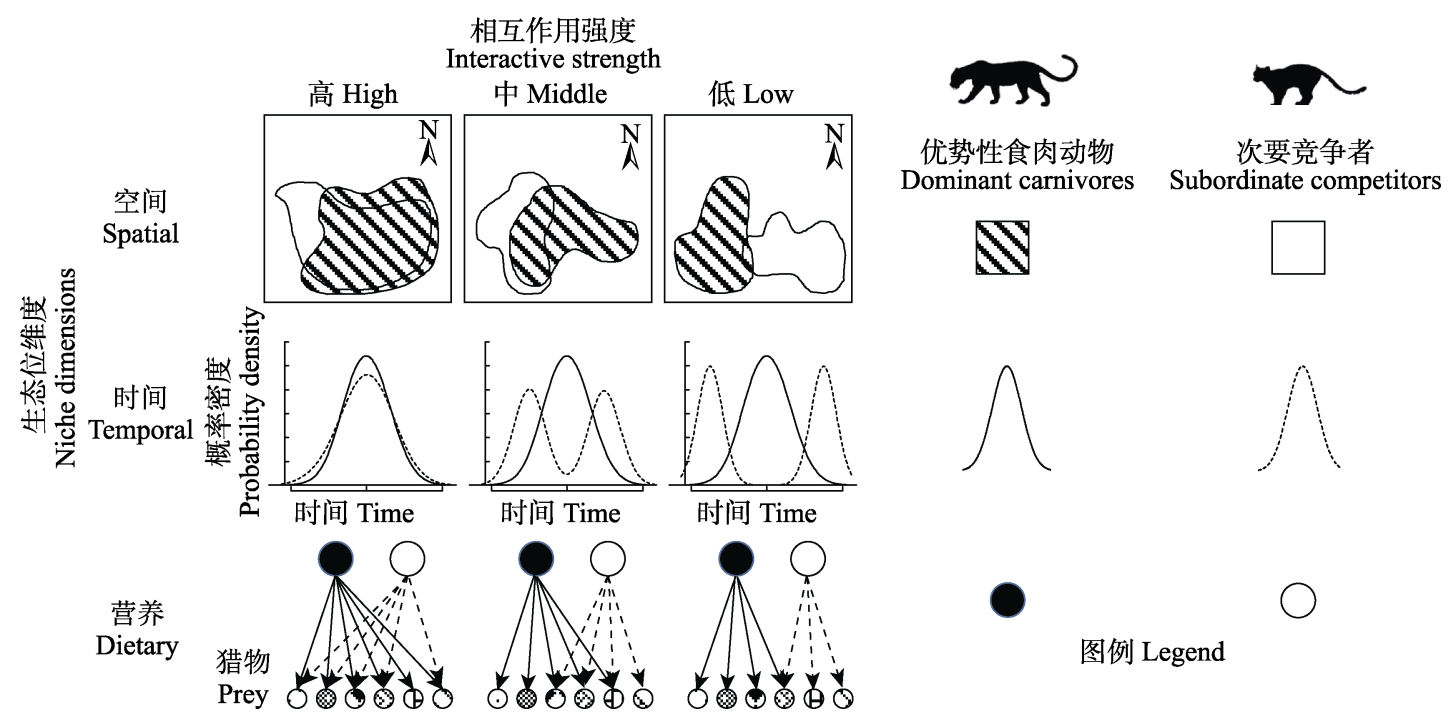

图1＼cjkstart优势性食肉动物和次要竞争者需要在空间、时间和营养等生态位维度分化以促进共存

Fig. 1 Dominant carnivores and subordinate competitors need to segregate among spatial, temporal and dietary niche dimensions to promote coexistence

用强度，包括干涉型竞争(interference competition, 直接相遇与杀戮)和资源利用型竞争 (exploitation competition，对共同的猎物资源的利用) (Kronfeld-Schor \& Dayan, 2003)。食肉动物在空间、时 间以及营养生态位上的可塑性和适应是一种减缓竞 争的演化结果(图1), 可以促进不同物种的共存(Sæbø, 2016)。

本文从空间、时间和营养生态位这 3 个维度综 述了食肉动物区域共存机制, 指出现有食肉动物相 互作用研究中存在的局限, 并提出了食肉动物共存 研究的发展方向。本文主要围绕以下 3 个方面展开: (1)哪些因素影响食肉动物在空间、时间和营养生态 位上的分化并调节物种共存? (2)在气候变化背景下, 促进食肉动物区域共存的关键因素有哪些? (3)食肉 动物区域共存有哪些亟需解决的问题? 以期促进 对于食肉动物区域共存的理解, 并为今后相关研究 方向的确立与方案设计提供参考。

\section{1 空间生态位竞争与共存}

空间生态位是理解食肉动物区域共存和相互 作用的基础。物种只有在一定空间范围内共存, 才 可能在时间和营养等生态位维度上潜在地发生相 互作用(Farris et al, 2020)。大量研究证明, 空间使用 差异能有效促进物种共存, 但在全球尺度上, 具有 相似生态特征的物种并不会产生完全的空间竞争
性排除, 反而会选择资源相似的空间来促进共存 (Davis et al, 2018)。大型食肉动物也因此发生区域 性聚集而形成全球的8个热点分布区(Ripple et al, 2014)。集团内捕食理论(Intraguild Predation Theory, IGPT)很好地解释了食肉动物间的作用关系, 强调 在集团内不同食肉动物之间同时存在资源利用型 竞争与干涉型竞争(Holt \& Polis, 1997)。集团内捕食 理论主要表现为同域分布的食肉动物为了争夺资 源会进行一系列恐吓、杀翏甚至生态位排除 (Palomares \& Caro, 1999; Vanak et al, 2013)。在区域 尺度, 斑块资源的质量与分布对优势性食肉动物的 空间分布起决定性作用(Karanth et al, 2017), 而体 型较小的次要竞争者除了需要考虑栖息地质量因 素外, 还要承受来自优势性食肉动物的空间压制 (Mondal et al, 2012)。例如, 在南亚区域, 虎 (Panthera tigris)作为优势物种占据了猎物资源丰富 的优势生境, 把豹排挤到周边的劣质生境中(Odden et al, 2010; Harihar et al, 2011)。优势性食肉动物除 了直接对次要竞争者进行空间排除外, 也会通过遗 留足迹、粪便、尿液等痕迹信息来间接地迫使次要 竞争者改变行为和活动空间(Torretta et al, 2016; Haswell et al, 2018)。这种间接作用符合代价收益原 理, 以尽可能减少食肉动物因集团内捕食作用带来 的适宜性降低。

食肉动物的空间关系会受到体型、性别、种群 
密度、活动节律和空间定位感知能力等生物学因素 的调节。Davis等(2018)对全球768例食肉动物区域 共存研究的整合分析结果表明, 大型食肉动物相较 于小型食肉动物更容易因种间竞争而产生空间躲 避。此外, 活动节律差异较大的物种更容易空间共 存(de Satgé et al, 2017)。在美国, 雌性的黑足鼠 (Mustela nigripes) 只会对雌性的美洲獾 (Taxidea taxus)表现出空间回避, 而对雄性则几乎不回避 (Grassel et al, 2015)。因此, 不同性别美洲獾空间分 布的差异导致了黑足鼣被捕食风险的空间异质性。 种群密度对食肉动物的空间作用调节表现出不对 称性：当次要竞争者密度增大时, 优势性食肉动物 会增大对栖息地的利用强度，比如增大日活动距离 或家域面积; 当优势性食肉动物密度增大时, 次要 竞争者会减少对优质生境的利用, 转向较为安全的 劣质生境(St-Pierre et al, 2006)。此外, 有研究发现食 肉动物还表现出跟踪和感知对方空间位置的能力, 可以与竞争者保持最小间隔距离, 以此来减缓种间 竞争, 表明食肉动物间风险躲避是一个反应过程而 不是预测过程(López-Bao et al, 2016)。

隐蔽所的使用是次要竞争者在空间上躲避优 势性食肉动物的有效方式(Salo et al, 2008)。空间异 质性较高的栖息地可以提供多种生境类型，满足不 同食肉动物生境选择的需求, 同时也增加了潜在的 隐蔽所来降低种间相互作用以促进区域共存(Finke \& Denno, 2006; McGee et al, 2006)。在东北亚, 东北 虎(P. t. altaica) 偏好在山谷巡视领地, 而东北豹 $(P . p$. orientalis)偏好在山脊活动, 生境选择的差异是这两 种大型猫科动物在有限空间内共存的重要因素 $(\mathrm{Li}$ et al, 2019)。在山地生态系统中, 海拔是空间异质性 的主要体现, 对食肉动物区域共存具有重要意义。 例如, 在苏门答腊岛, 苏门答腊虎(P. t. sumatrae)、 巽他云豹(Neofelis diardi)、亚洲金猫(Catopuma temminckii)、云猫 (Pardofelis marmorata) 及豹猫 (Prionailurus bengalensis)具有不同的海拔利用偏好, 使得这 5 种猫科动物能够在 $6-460 \mathrm{~m}$ 的海拔区间内 共存(Sunarto et al, 2015)。海拔因素也有利于高密度 的同种食肉动物的区域共存。例如, 不同波斯豹 $(P$. p. saxicolor)个体的家域能沿海拔梯度产生分化而 降低种内竞争(Farhadinia et al, 2019)。微生境空间分 离和行为特征也是促进食肉动物区域共存的重要 因素, 特别是多种小型食肉动物具有的穴居性或树
栖性等特点, 可以有效避免主要在地面活动的优势 性食肉动物的空间压制。如狗獾属(Meles)动物通过 长时间的地下活动来避免大型食肉动物的威胁 (Roper, 1992); 香鼠(Mustela altaica)在地面对赤狐 (Vulpes vulpes)并没有表现出明显的趋近或趋避，却 可以躲进岩石缝隙或者地洞中来躲避赤狐的捕食 (Bischof et al, 2014)。

猎物可以沿食物链的上行作用(bottom-up)对食 肉动物产生影响(Li et al, 2017)。猎物的丰富度、物 种组成、可获得性和空间分布在调控食肉动物空间 分布和区域共存上可以起到决定性作用(Odden et al, 2010; Steinmetz et al, 2013; Carter et al, 2015)。而 且在决定食肉动物空间分布与区域共存方面，猎物 因素可能比食肉动物种间作用更为重要(Santos et al, 2019)。一方面, 较充足的食物资源会缓解食肉动物 空间生态位竞争。例如, 在印度纳加拉霍雷国家公 园(Nagarahole National Park), 因为猎物资源丰富, 虎并没有对豹产生明显的空间排除，而是在更精细 的空间尺度以及行为上对豹进行限制(Karanth \& Sunquist, 2000)。当猎物资源较贫乏时，优势性食肉 动物会通过增加活动频率来提高对猎物的搜索强 度, 继而可能会提高与次要捕食者的相遇概率, 导 致对后者的捕食概率增加。另一方面，不同食肉动 物对猎物利用的协同作用也可能影响食肉动物的 空间关系(Prugh \& Sivy, 2020)。在美国大黄石地区， 赤狐作为小型食肉动物很难捕食大型的有蹄类猎 物, 但会选择利用美洲狮(Puma concolor)密度较高 的区域，主动搜寻和取食美洲狮捕获猎物的残骸， 通过美洲狮的协助增加自己的食物收益(O’Malley et al, 2018)。

人类活动可以对食肉动物的空间活动带来严 重干扰, 被认为是影响野生动物产生景观恐惧 (landscape of fear)的重要因素(Støen et al, 2015)。人 类干扰导致的栖息地类型转变和资源衰退很大程 度上重塑了全球食肉动物的空间分布格局(Ripple et al, 2014)。栖息地持续丧失与生境质量不断下降 使处于食物链顶端的大型食肉动物相继灭绝，继而 引发中级捕食者释放 (meso-carniviore release; Brashares et al, 2010), 从根本上改变了食肉动物固 有的空间共存模式。在区域尺度，栖息地的破碎化 与斑块化使食肉动物往往分布在有限空间内，进而 抑制了空间上的进一步分化(Geldmann et al, 2014)。 
随着人类城镇化水平的不断提高, 食肉动物的家域 面积不断被压缩(Šálek et al, 2015), 迫使食肉动物 增加对次优生境的利用强度(Wilmers et al, 2013), 增加了它们获取优质食物资源的难度。考虑到人类 活动可以潜在地改变食肉动物的行为 (Oriol-Cotterill et al, 2015), 同域分布的竞争性食肉 动物可以在人类主导的系统中, 通过对人类活动 (例如人类频繁访问或者城市化发展)忍受的差异性 反应来重塑食肉动物之间的关系(Polis \& Holt, 1992)。大型食肉动物通常对人类活动表现出较为严 格的空间回避, 而小型食肉动物则偏好在居民密集 的区域活动(Nickel et al, 2020)。在美国加利福尼亚 地区, 美洲狮对居民点表现出空间回避, 而斑臭鼠 (Spilogale gracilis)等小型动物则把居民点作为躲避 美洲狮的避难所(Wang et al, 2015)。因人类干预导致 食肉动物空间生态位重叠度的降低可以被理解为 一种警告信号, 表明食肉动物之间原有的种间关系 已被破坏(Sévêque et al, 2020)。

此外, 人类活动导致的外来物种入侵会对本地 食肉动物产生空间压制。家狗(Canis lupus familiaris) 和家牛(Bos primigenius taurus)作为全球最常见的 伴人入侵物种, 会对本土食肉动物产生严重的空间 排除, 并潜在影响食肉动物的种间关系(Farris et al, 2016; Li et al, 2019; Mella-Méndez et al, 2019a)。狗 可作为捕食者、猎物、竞争者、病源和疾病传播者 等不同角色, 通过多种途径对野生食肉动物产生影 响, 并能通过与野生食肉动物分享传染性疾病与寄 生虫而重塑食肉动物种间关系(Vanak \& Gompper, 2009; Silva-Rodríguez \& Sieving, 2012)。在流浪狗密 度较高、大型食肉动物匮乏的城市与近郊生态系统 中, 狗可能对小型食肉动物造成较大的生态压制 (Vanak \& Gompper, 2009; Yen et al, 2019)。但专职的 畜牧看护犬可以有效减低野生大型食肉动物对家 畜的捕食, 降低因家畜损失带来的人兽冲突强度, 促进人与野生食肉动物的区域共存(Spencer et al, 2020)。长期以来, 家畜放牧已成为全球最主要的土 地利用方式之一, 能导致野生动物群落多样性下降 和物种构成发生改变(Schieltz \& Rubenstein, 2016)。 例如, 散放家牛会降低有蹄类猎物多度和空间分布, 进而通过营养级联效应(trophic cascading effect)影 响食肉动物的种间竞争关系(Filazzola et al, 2020)。 在亚洲, 许多高强度放牧的地区, 高密度的家畜会
导致野生猎物资源的下降和生境的丧失, 使得虎和 豹的空间竞争加剧, 甚至导致局域种群的消失 (Ramakrishnan et al, 1999; Wang et al, 2016; Li et al, 2019)。

\section{时间生态位竞争与共存}

时间生态位是生态位理论中物种生态位构建 的重要组成部分(Hutchinson, 1957, 1959)。时间也可 以看作一种限制性资源, 为食肉动物提供了一种行 为改变维度来促进共存(Halle, 2000)。动物的活动节 律是描述其时间生态位的主要方式, 是描述一天中 动物怎样分配活动时间的一种量化方式(Schoener, 1974); 活动节律受到生物机体内在调节与外部因 素的共同影响, 以适应自然环境的周期性变化 (Kronfeld-Schor \& Dayan, 2003)。目前有关食肉动物 时间生态位维度关系的研究主要集中于日活动节 律, 或是在其他时间尺度探究日活动关系的差异 性。在群落水平, 理解同域分布物种的时间分配 (time budget)对理解物种共存机制具有重要作用 (Carothers \& Jaksić, 1984; Kronfeld-Schor \& Dayan, 2003)。野生动物的活动节律很容易受到当地环境影 响, 在生态研究和生物多样性保护中, 生物类和非 生物类的因素对动物活动节律和时间生态位重叠 的影响都是重要的问题(Halle, 2000)。很多研究表明, 捕猎(Di Bitetti et al, 2010)、人类活动(Wang et al, 2015; Ngoprasert et al, 2017)、捕食者控制(Brook et al, 2012) 、土地类型变化(Ramesh et al, 2012)和外来 物种入侵(Gerber et al, 2012; Zapata-Rios \& Branch, 2016)等都会改变动物的活动节律和种间关系。因此 在制定保护决策时, 除了需要考虑到干扰因素可能 影响同域物种的空间共存, 还应考虑各因素对物种 时间生态位关系的影响(Frey et al, 2017)。

食肉动物在时间和空间生态位上的活动差异 是降低竞争强度的重要途径(Karanth et al, 2017)。相 较于空间生态位, 时间生态位分化具有弹性大、灵 活度高的特点, 可以有效降低很多同域分布且具有 相似生态特征的食肉动物的集团内捕食风险。当食 肉动物在空间、营养和功能性状等方面具有较高相 似性时, 活动节律的差异可以有效促进区域共存 (Andersen et al, 2020)。如墨西哥的灰狐(Urocyon cinereoargenteus)与长鼻浣熊(Nasua narica)在食性 和栖息地使用方面具有较高相似性, 但在时间生态 
位上产生分化(Gómez-Ortiz et al, 2019)。东北虎与东 北豹种群因适宜生境有限, 很难在空间生态位上产 生显著分化, 因而时间生态位分化就成为促进二者 区域共存的重要基础(Li et al, 2019)。对新热带区域 同域分布猫科动物的研究发现, 形态特征越相似的 物种，时间生态位的分化越明显(Di Bitetti et al, 2010)。因此，同域分布物种的时间生态位分化可以 作为它们在其他生态位维度上差异性缺失的一种 补充(Frey et al, 2017)。

同域分布食肉动物的物种丰度(species richness) 可能通过影响时间生态位对食肉动物的稳定共存 起到重要作用。研究发现, 小型食肉动物群落的物 种丰度与每对物种的时间生态位平均重叠度呈显 著负相关(Frey et al, 2017)。当在有限区域内同域分 布多种食肉动物时，种间压制导致的时间生态位分 化更为明显。Monterroso等(2014)对非洲同域分布的 狮(Panthera leo)、豹、猎豹(Acinonyx jubatus)、斑 点镾狗(Crocuta crocuta)、非洲野狗(Lycaon pictus) 等6种食肉动物的时间生态位分析发现, 次要竞争 者(非洲野狗与猎豹)的活动明显避开其他优势性食 肉动物(Hayward \& Slotow, 2009)。在这个食肉动物 群落中, 优势性食肉动物主要为夜行性与晨昏活动, 而次要竞争者则表现为昼行性活动, 以躱避来自优 势性物种的干扰性压制, 并防止优势性物种前来抢 夺捕获的猎物(偷窃寄生行为)。

优势性食肉动物的活动节律很大程度上受到 猎物活动模式的驱动, 不同猎物物种活动节律的差 异是调节食肉动物活动节律差异的主要因素。次要 竞争者在时间生态位上表现出较高的灵活性与容 忍性, 其活动节律除受到猎物驱动外, 还要受优势 性食肉动物活动和环境因素的调节。在印度的西高 止山脉, 虎与其主要猎物水鹿(Rusa unicolor)的活 动节律具有较高的重叠度, 豹针对时间生态位所受 的胁迫表现出较高的容忍性, 从而能够更好地兼顾 各种资源的综合利用(Ramesh et al, 2012)。在虎被人 为引入之前或者没有虎分布的区域, 豹表现出夜行 性和晨昏活动的特点; 虎被重引入以后或者在虎已 经存在的区域, 豹则变为昼行性活动, 以降低与虎 活动的重叠(Mondal et al, 2012; Steinmetz et al, 2013)。在非洲, 猎豹的昼行性节律使其可以有效避 免与夜行性的狮之间的冲突; 但在满月期间, 猎豹 会增大夜间的活动频率, 因为较强的月光会提高其
夜晚捕猎的成功率，表明月光强度对猎豹在受食需 求和捕食风险权衡中具有调节作用(Broekhuis et al, 2014)。即使次要竞争者为躲避优势性食肉动物而发 生活动节律的改变, 很多次要竞争者仍在时间或空 间等维度与主要捕食对象保持较高的生态位重叠 (Bischof et al, 2014; Li et al, 2019)。次要竞争者的时 间生态位选择同时满足了对优势性物种的躲避和 对最优猎物受食的需求, 最大限度避免了因优势性 食肉动物生态压制而造成的适合度降低。

人类活动和栖息地景观的改变可以对食肉动 物活动节律和时间生态位关系产生重要影响。野生 哺乳动物在人类控制区域会增加夜间活动的强度 已成为全球性现象(Wang et al, 2015; Gaynor et al, 2018), 而在城市中心则会表现出完全的夜行性 (Ditchkoff et al, 2006)。但人类干扰移除后，食肉动 物又会逐渐恢复其原有的活动节律 (Kitchen et al, 2000)。食肉动物也表现出对昼行性散放家养动物 (例如家牛和家狗)的躲避(Mella-Méndez et al, 2019b; Yen et al, 2019)。大量的人类活动会对食肉动物造成 压倒性的时间压制，迫使食肉动物之间的时间生态 位重叠加大，继而加剧种间竞争(Wang et al, 2015)。

\section{营养生态位竞争与共存}

营养生态位是野生动物生态位的一个重要属 性, 能够影响动物在生态系统及食物网中的功能。 比较同域分布物种的食性可以揭示不同物种之间 营养生态位的重叠程度，并以此作为物种间潜在竞 争强度的量度之一(du Preez et al, 2017)。食物资源 的分割可以有效降低食肉动物的种间竞争(Lovari et al, 2015)。猎物丰富度、种间竞争、系统发育等 多种因素都能影响食肉动物的受食差异(Radloff \& Du Toit, 2004; Andheria et al, 2007), 特别是食肉动 物的体型差异可以起到决定性作用(Gómez-Ortiz et al，2015)。捕食者体型与猎物体型一般成正相关关 系(Scharf et al, 2000)。许多大型食肉动物表现为专 性肉食性, 主要以大中型哺乳动物为食(Simcharoen et $\mathrm{al}, 2018)$, 而小型食肉动物则大多属于机会主义 捕食者，可以捕食小型哺乳动物、鸟类、两栖类、 爬行类、无脊椎动物并取食部分植物，表现出一定 的杂食性并具有较宽的营养生态位(张雨嘉等, 2011; Choi et al, 2015; Elmeros et al, 2018)。

食肉动物营养生态位宽度与体重一般呈单峰 
关系, 研究发现体重约为 $2.5 \mathrm{~kg}$ 时食肉动物表现出 最大的营养生态位宽度(Lanszki et al, 2019)。食肉 动 物物种之间的营养生态位重叠度随着种间体重差 异的增大而呈现非线性减少趋势; 体重差异最小 (约为 $0.01 \mathrm{~kg}$ ) 时, 预测营养生态位重叠度为 $62 \%$; 体重差异最大(160 kg)时, 预测的营养生态位重叠 度为 $12 \%$ (Lanszki et al, 2019)。同域分布的大型与小 型食肉动物之间一般不存在高度的食性重叠 (Gómez-Ortiz et al, 2015), 而体型相似的食肉动物 之间因为捕食相似的猎物而更可能发生激烈的种 间竞争甚至出现杀翏行为(Donadio \& Buskirk, 2006)。因此食肉动物需要在猎物的物种(Karanth \& Sunquist, 2000)、体型 (Gittleman, 1985)、年龄 (Karanth \& Sunquist, 1995)、取食比例(Andheria et al, 2007)和捕食地点(Palomares et al, 1996)等方面产生 分化, 以缓解竞争来促进共存。如在南亚区域, 虎 作为优势性物种表现出对野猪(Sus scrofa)、水鹿、 印度野牛(Bos gaurus)等大型猎物的偏好(Andheria et al, 2007; Wang \& MacDonald, 2009; Kerley et al, 2015)。同域分布的豹则在选择体型稍小的白斑鹿 (Axis axis)、豚鹿 (A. porcinus)的同时, 还捕食劣质生 境中的家畜等小型猎物, 表现出更为宽泛的食谱 (Mondal et al, 2012; Lovari et al, 2015; Pokheral \& Wegge, 2019)。在欧洲北部, 同域分布的貉 (Nyctereutes procyonoides)、欧亚狗獾(Meles meles) 以及赤狐 3 种小型食肉动物有着相似的食物谱, 但 是却在各成分的组成比例上具有明显差异: 貉更多 地捕食啮齿类等小型哺乳动物, 赤狐则偏向于捕食 兔形目等体型稍大的哺乳动物, 欧亚狗獾则倾向于 捕食无脊椎动物(Kauhala et al, 1998)。

偷窃寄生行为是食肉动物间资源竞争的重要 方式, 是一种食肉动物通过偷窃或抢夺的方式从另 一种食肉动物中获取食物等资源以增加收益的行 为(Nishimura, 2010)。食肉动物通过偷窃寄生可以用 较少的投入获得巨大的收益(Cusack et al, 2017)。在 非洲, 豹捕食的猎物有 $21 \%$ 会遭到狮、斑点镾狗和 其他豹个体的抢夺或偷窃, 而较大的“猎物/捕食者” 体型比能显著促进偷窃寄生行为的发生(Balme et al, 2017)。当猎物/捕食者体型比为2时, 豹捕食的猎物 有 $50 \%$ 的概率被抢夺或偷窃, 因此导致豹在选择捕 猎对象时, 可能会选择比最优受食理论所预测的体 型更小的猎物, 以此作为应对偷窃寄生行为与维持
自身能量需求之间的一个权衡。偷窃寄生行为会降 低猎物被抢夺或偷窃的食肉动物的适合度, 比如导 致雌豹的繁殖率降低，因此会促使这些食肉动物采 取藏食行为来躲避同域分布的偷窃寄生者, 增加自 身进食量(Balme et al, 2017)。比如豹通常会把猎物 藏到树上来避免被抢夺和偷窃。优势性食肉动物通 过捕食为生态系统提供猎物残骸和腐肉, 客观上可 以作为一种生态系统服务, 而作为清道夫的次要竞 争者则会促进这种资源在景观上的扩散, 并通过猎 物残骸和腐肉的不均匀分布而增加景观的异质性, 食肉动物对猎物残骸和腐肉产生与消耗的协同作 用会促进区域共存(O’Malley et al, 2018)。

系统发育对食肉动物营养生态位关系也具有 调控作用, 主要表现在具有共同演化起源的同一类 群内的物种在体型大小、形态特征和活动方式上比 非同类物种具有更高的相似性, 因此会有更加一致 的猎物偏好，从而增加资源利用型竞争(Donadio \& Buskirk, 2006)。

猎物的多度(abundance)和物种丰度(richness)可 以对食肉动物营养生态位关系产生不同影响。猎物 多度越高, 同域分布食肉动物的食性越相似 (Karanth \& Sunquist, 1995; Andheria et al, 2007)。较 高的猎物多度可以使食肉动物更容易获得高能量 收益的猎物从而降低资源利用型竞争以促进共存。 在泰国, 当虎、豹和豹(Cuon alpinus)共存于猎物资 源较差的生境时, 各物种的营养生态位宽度显著大 于猎物资源丰富的区域, 而物种间的营养生态位重 叠度则低于猎物资源丰富的区域(Steinmetz et al, 2020)。同时, 较高的猎物物种丰度使得生态系统中 的猎物种群在空间分布、活动时间和体型大小上表 现出更大的异质性, 为不同种类的食肉动物物种提 供了更多的选择可能, 有利于食肉动物共存 (Ramesh et al, 2012)。因此维持生态系统中猎物群落 的多度和物种丰度对促进食肉动物区域共存有重 要意义。

食肉动物间的营养生态位关系还会受到集团 内捕食作用的调控。优势性食肉动物对次要竞争者 的捕杀一般有两个动机: 一是把次要竞争者作为食 物, 二是减少次要竞争者密度以降低种间竞争强度 (Ritchie \& Johnson, 2009)。当次要竞争者与优势性 食肉动物体型大小很接近时, 它们可能捕食共同的 猎物, 彼此是重要的生态竞争者; 当它们体型差异 
较大时, 次要竞争者更容易受到优势性食肉动物伤 害。优势性食肉动物对次要竞争者发起捕食攻击的 可能性由二者体型大小比例决定：比例小于 2 时, 优势性食肉动物更容易在攻击中受到次要竞争者 反击的伤害; 比例为2-5.4时, 优势性食肉动物最可 能对次要竞争者发起捕食性攻击; 比例大于 5.4 时, 优势性食肉动物则难以得到明显的生态收益 (Donadio \& Buskirk, 2006)。在评估食肉动物间的捕 食作用时, 很重要的一点是确定种间捕杀造成的死 亡是补偿性的 (compensatory) 还是附加性的 (additive)。如果次要竞争者受到优势性食肉动物的 捕杀只改变了影响种群稳定的某一因子(例如优势 性食肉动物只捕杀老幼病残个体而缓解次要竞争 者的种内竞争), 此时捕杀所造成的死亡率会对次 要竞争者种群造成一种持续的积极影响, 有利于后 者的种群稳定与种间共存(补偿性捕杀); 而当种间 捕杀不只针对次要竞争者的弱势个体, 此时种间捕 杀成为附加性的致死因素而导致次要竞争者种群 数量持续下降甚至灭绝, 则不利于食肉动物共存 (Ralls \& White, 1995)。

气候变化对食肉动物营养生态位关系的影响 主要体现在通过改变栖息地质量和猎物分布, 继而 可能对食肉动物的食性选择和竞争产生间接影响 (Carroll, 2007)。此外，人类控制景观中存在大量人 造食物和城市垃圾, 也可能造成各物种食性和食物 组成的改变。例如, 印度城市景观中生活的赤狐大 量利用人造食物, 这一现象在食物短缺的冬季尤为 明显(Ghoshal, 2011)。

\section{4 空间、时间和营养生态位协同作用}

空间、时间和营养生态位在促进物种区域共存 进程中相互协调, 互为补充。集团内捕食理论很好 地解释了空间、时间和营养生态位在促进食肉动物 区域共存的协同作用。优势性食肉动物对次要竞争 者施加生态压迫主要表现在两个方面: 一是把次要 竞争者作为猎物直接捕食, 二是增加次要竞争者的 生态恐惧以减少其对共同资源(例如猎物)的竞争, 一般以后者为主要作用。很多情况下，优势性食肉 动物会杀死次要竞争者但并不取食, 同时次要竞争 者因生态恐惧而降低与优势性食肉动物在空间、时 间和营养生态位的重叠(Ritchie \& Johnson, 2009)。 食肉动物需要对猎物可获得性、被捕食风险及人类
干扰等诸多因素进行权衡, 选择最佳行为策略以取 得最大收益(Sévêque et al, 2020)。因此, 食肉动物在 一个生态位维度的行为方式会受到其他生态位维 度行为策略的影响, 并与整个环境背景相契合。在 尼泊尔的奇旺国家公园(Chitwan National Park)共存 的虎与豹, 在空间生态位上, 虎会把豹排挤到公园 外猎物资源较差的劣质生境; 在营养生态位上, 虎 主要捕食水鹿等大型野生猎物, 而豹只能捕食小型 猎物和家畜(Bhattarai \& Kindlmann, 2012); 在时间 生态位上, 豹在国家公园内部活动时会进行妥协, 以昼行性活动为主来降低与虎的干涉型竞争, 而在 公园外豹则可以保持夜行性来增加与猎物的时间 生态位重叠, 同时降低与人类的相遇概率(Carter et al, 2015)。空间、时间和营养生态位可以协同作用 达成一种动态平衡状态。当外界环境改变时 (如外来 物种入侵或顶级捕食者灭亡), 食肉动物可能采取 以下策略：(1)保持原有生态位策略不变; (2)改变生 态位策略以降低环境变化带来的损失并达到新的 平衡状态; (3)改变生态位策略并达到应对环境变化 的过渡状态(Farris et al, 2020)。不同策略的选择依 然受到代价收益的调控。

\section{我国食肉动物竞争共存研究}

我国是全球食肉动物物种多样性最为丰富的 国家之一, 涉及多个食肉动物多样性分布热点区域 (Ripple et al, 2014)。蒋志刚等(2017)记录我国共有 食肉目哺乳动物 10 科 40 属63种, 其中陆生食肉目哺 乳动物 8 科 58 种; 根据野外记录, 近期确认在我国 有分布的陆生食肉目哺乳动物共计 8 科 51 种 (刘少英 和吴毅, 2019), 约占全球陆生食肉目哺乳动物物种 总数的 $20 \%$ 。长期以来人类社会的生产生活活动对 自然生境的破坏导致我国食肉动物栖息地大量丧 失, 种群数量和分布范围剧烈缩小, 潜在种间关系 发生改变(Li ZL et al, 2019; Li S et al, 2020)。目前, 我国的食肉动物研究与保护主要以虎、雪豹、豹等 明星物种为引领, 并在长白山、完达山、秦岭和西 南山地等区域开展了较为系统的食肉动物研究 (Wang TM et al, 2017; Wang F et al, 2018)。但我国对 食肉动物竞争与共存的研究仍不够系统与深入, 大 多数食肉动物特别是小型食肉动物没有得到足够 关注。使用谷歌学术以 “物种名 + China + interaction”为关键词检索2000-2020年发表的文献, 
发现研究者对我国分布的 $63.3 \%$ 的大型食肉动物开 展过不同程度的生态位竞争与共存研究, 而这一比 例在我国小型食肉动物中只占 $28.6 \%$ 。豹猫、赤狐、 亚洲狗獾(Meles leucurus)、猪獾(Arctonyx collaris)、 果子狸(Paguma larvata)、黄喉貂(Martes flavigula)、 黄鼠(Mustela sibirica) 等广布物种具有较高的科研 关注度(Hua et al, 2020; Zhao et al, 2020)。如Bu等 (2016)使用双物种占域模型证明了西南山地果子狸 与猪獾之间负的空间关系。张逦嘉等(2011)利用粪 便内容物分析了内蒙古赤狐、亚洲狗獾、香鼠和豹 猫的食性重叠程度。而小型食肉动物中的临科、灵 猫科、獴科和一些小型猫科动物, 如野猫 (Felis silvestris)、荒漠猫 (F. bieti)等, 则存在较大的研究空 白。许多小型食肉动物物种具有种群数量少、分布 范围狭窄等特点, 需要今后给予重点关注。关注程 度较高的大型食肉动物多为生物多样性保护中的 旗舰物种, 但针对少数旗舰物种而设置的保护策略 与管理计划不一定能够充分满足其他同域物种的 生态需求(Shen et al, 2020), 容易造成保护工作的片 面性(Li et al, 2020)。结合物种共存理论的群落水平 研究和大型动物区系的物种共存机制研究应成为 我国未来食肉动物共存研究的重点。此外, 我国现 有的食肉动物研究多为应用型研究, 针对种间交互 作用及物种区域共存机制的理论研究和方法创新 也应成为未来关注的重点方向。

我国对城市景观中食肉动物竞争与共存的研 究较为贵乏。城市野生动物会在食性、活动行为等 方面发生改变以适应城市化特征, 因而与自然景观 中的野生动物种群表现出不同的生态响应(Santini et al, 2019)。国外已有部分研究对食肉动物在城市 景观中的竞争与共存开展了较为系统的探索 (Gallo et al, 2017; Mueller et al, 2018), 例如阐明了北美城 市中绿岛斑块的可获得性对短尾猫(Lynx rufus)和郊 狼(Canis latrans)的竞争共存具有重要的调节作用 (Parsons et al, 2019)。我国城市景观中通常缺乏大面 积自然或半自然栖息地, 因而难以承载大型食肉动 物种群。但最近的报道表明, 随着我国生态环境的 改善, 黄鼠由、貉等小型食肉动物正逐步在部分城市 景观中建立种群(http://www.xinhuanet.com/english/ 2020-03/25/C_138916301.htm)。在我国城市景观开 展食肉动物竞争与共存等研究, 能够加深对高强度 人类干扰下食肉动物群落组装与构建的理解, 同时
对疾病防控、入侵物种管理、城市建设与规划具有 重要意义(Gallo et al, 2017)。

\section{总结与展望}

猎物、栖息地和人类干扰等关键因素可以调节 食肉动物在空间、时间和营养生态位之间的相互作 用关系, 并直接或间接地影响食肉动物共存。同时, 食肉动物区域共存是演化过程中长期自然选择所 达到的相对稳定状态, 因此并没有单一的机理可以 全面解释不同动物群落的构建机制。食肉动物生态 位分离和区域共存需要与分布地背景相契合, 因此 需要在理解猎物丰富度和群落组成、栖息地资源质 量与分布、人类干扰类型与强度等背景特征的基础 上进行综合分析(Odden et al, 2010; Lovari et al, 2015)。共同适应是促进食肉动物区域共存的关键因 素, 食肉动物需要在演化过程中不断学习, 并调整 行为以应对环境、气候和人类干扰的变化(Carter \& Linnell, 2016)。共存是物种关系不断变化中的相对 稳定状态, 需要以动态和演化的视角去理解食肉动 物的区域共存。目前食肉动物竞争与共存研究多使 用生态位重叠程度表征相互作用强度(du Preez et al, 2017; Shamoon et al, 2017; Allen et al, 2018)。但生态 位重叠程度仅是一种表象, 并不能等价于食肉动物 相互作用强度。例如, 两种食肉动物高度的空间重 叠表明两者与随机状态相比更偏好使用同样的空 间, 而高度的时间重叠只表明食肉动物在同一个区 域更可能发生交互作用而不代表二者活动具有一 致性。每一个生态位维度上, 重叠的表象并不一定 是共存物种发生交互作用的结果 (Farris et al, 2020)。在考虑生态位重叠的同时, 更需要充分结合 详细的自然史、运动(家域)以及行为信息来对食肉 动物的区域共存进行解读。

由于理论方法与认知水平的限制, 食肉动物竞 争与共存研究长期存在关注尺度和维度单一、技术 手段落后、研究目标粗放等问题, 严重限制了对于 食肉动物群落组装与构建机制的理解。近年来, 红 外相机(O’Connell et al, 2010; 李晟等, 2014; 李晟, 2020)、环境DNA (Bohmann et al, 2014)、高通量测 序 (high-throughput sequencing, HTS) 与宏条形码 (Monterroso et al, 2019; 郡昕宁等, 2019)等数据采 集技术和占域模型以及多物种模拟方法的发展 (Richmond et al, 2010; Tobler et al, 2019), 极大地促 
进了食肉动物种间相互作用研究的发展。未来的食 肉动物竞争与共存研究在继续围绕空间、时间和营 养三个生态位维度开展的同时, 还应鼓励多视角的 理论与技术创新。在科学问题阐述上既要细致明确, 又要做到多角度整合(例如从多尺度和多维度探究 竞争与共存)。鼓励应用控制性实验探究食肉动物的 种间相互作用, 从而推动食肉动物竞争与共存研究 的精细化、精准化和全面化发展。今后, 食肉动物 区域共存研究应着重在以下各方面开展深入探索 并注意以下问题，总结如下:

(1)在空间生态位研究中明确区分种间关系与 共同生境偏好。已有的大多数空间生态位研究中, 没有很好地区分种间关系与共同生境偏好之间的 关系。不同物种对栖息地共同选择所产生的表象, 有可能会潜在地导致对物种空间关系的错误判断 (Farris et al, 2020)。例如, 在泰国, 食蟹獴(Herpestes urva)与虎都选择相似的溪流、河床生境, 但其原因 并不相同: 食蟹獴是为了捕食半水生的猎物, 而虎 则是利用该生境来巡护领地, 因此产生了两者空间 分布上存在正相关关系的表象(Chutipong et al, 2017)。与之类似, 捕食者之间的空间排斥作用也可 能只是反映了不同的生境选择。研究者需要充分了 解食肉动物的受食、集群、繁殖、活动规律等生物 学和生活史信息, 以物种的生态需求而不是模型统 计为导向, 从而精准探究食肉动物的生物学关系。

(2)在时间生态位研究中应考虑不完美探测的 影响。已有研究在探究物种时间生态位关系时, 往 往没有考虑不完美探测(即动物在某位点存在但调 查中未被记录到)的问题。如果在分析中忽视不完美 探测的实际情况, 可导致对物种活动节律的估计产 生较大偏差(Lashley et al, 2018)。例如在基于红外相 机技术 (camera-trapping)开展的食肉动物调查与研 究中, 较低的环境温度可导致红外相机触发延迟甚 至漏拍, 这一现象在高纬度和高海拔区域的冬季监 测中尤为明显。食肉动物大多为夜行性和晨昏活动, 这些特点使得在时间生态位分析中加入对不完美 探测的考虑尤为重要。今后, 在对时间生态位的描 述中, 需要在方案设计中充分考虑不完美探测所带 来的可能影响, 开发相关技术对其数值进行量化评 估并纳入整体分析框架, 以对目标物种的时间生态 位进行更可靠、更全面的评估(Ait Kaci Azzou et al, 2019)。
(3)在营养生态位研究中充分发挥分子生物学 方法与技术的优势。分子生物学方法在食肉动物营 养生态学研究中的应用需要加强。虽然高通量测序 技术近年来快速发展, 但基于毛发、骨骼等粪便内 容物的传统形态学分析法仍在食肉动物营养生态 学研究中占较大比例, 而使用分子生物学技术进行 食肉动物食性分析的研究只占到全部食性研究数 量的8\% (Monterroso et al, 2019)。传统的粪便内容物 形态学分析法具有较高的假阳性(把其他物种的成 分错认为目标物种)和假阴性(漏掉目标物种或把目 标物种错认为非目标物种), 可能对食性分析结果 造成较大的误差和误判 (Lonsinger et al, 2015; Mumma et al, 2016)。今后的研究重点应聚焦于分子 生物学方法的普及与创新, 特别是对杂食性食肉动 物的食性分析(邵昕宁等, 2019), 以更为精准、可靠 的数据定量化地描述食肉动物的营养生态位以及 物种之间的营养生态位重叠程度。

(4)积极开展食肉动物区域共存与生态位重叠 的多维度、多尺度动态研究。目前多数研究独立地 从空间、时间和营养生态位探究食肉动物种间生态 关系，能够同时从时间-空间、空间-营养、时间-营 养等多维度探究物种关系的研究严重匮乏(Cusack et al, 2017; Karanth et al, 2017)。从单一生态位维度 去考虑物种关系势必会造成信息的丢失以及结果 的片面性。这种情况的出现主要是受限于数据采集 与分析方法, 目前跨生态位的统计方法创新性与稳 健性不足(Farris et al, 2020), 各监测体系之间也缺 乏有效连通。在今后的研究中, 要注重红外相机、 项圈追踪技术和食性分析等多维度方法的交互与 整合。

在研究尺度上, 现有的空间生态位研究往往尺 度单一, 缺乏从多尺度对食肉动物共存的理解。在 不同的空间尺度上, 影响食肉动物相互作用的因素 与途径、机制是不同的, 物种之间所表现出的相互 作用类型和方向也可能与研究的空间尺度相关, 即 存在尺度依赖效应。红外相机技术近年来快速发展, 并被作为食肉动物生态研究中的最有效手段之一 (O’Connell et al, 2010; 李晟等, 2014; 李治霖等, 2014; 李晟, 2020), 因此目前的研究多数聚焦于相 机位点尺度, 而在物种家域、斑块、景观乃至区域 尺度上的食肉动物共存研究相对较为缺乏。此外, 现有的时间生态位维度的研究主要集中于日活动 
尺度, 季节、年度等其他时间生态位尺度的研究同 样匮乏。食肉动物时间生态位相互作用强度可能存 在季节间的差异性(Vilella et al, 2020), 而从多时间 尺度探究食肉动物的交互与共存同样需要大量数 据的支持(Marinho et al, 2020)。食肉动物的种间关 系并不是固定不变的, 环境、人类干扰和食肉动物 种群结构在季节和年际间的变化均可能导致食肉 动物种间关系和生态位重叠程度的改变(Fedriani et al, 1999; Vanak et al, 2013; Petersen et al, 2019)。例如, 在北美地区, 赤狐与郊狼的空间生态位关系会因农 业景观的季节性变化而在夏季与冬季间表现出巨 大的差异(Gosselink et al, 2003); 生态旅游等季节性 人类活动的差异也可能潜在地改变食肉动物的生 态位作用关系与强度(Monterroso et al, 2014)。在食 肉动物区域共存研究中, 如果不考虑种间关系的动 态变化势必会造成认识的片面性(Sévêque et al, 2020), 而探究气候变化导致的食肉动物种间关系 的改变则需要长期的监测与积累。

(5)加强对食肉动物种间作用强度的直接量度。 食肉动物共存研究长期使用生态位重叠程度代表 种间作用强度, 而缺乏对食肉动物种间作用强度的 直接量度(Schoener, 1982)。生态位重叠程度并不能 完全表征物种间的作用强度, 存在有偏估计的风险 (Farris et al, 2020)。食肉动物种间作用强度可以通 过直接观测物种间争斗或对猎物的争夺频次进行 推断, 但该方法通常只适用于开阔生境, 无法用于 隐蔽度较高的生境(Seidensticker, 1976)。比较单位 面积内食肉动物种群密度变化的关系也可以对种 间作用强度进行推断, 但同样需要长期监测和大量 的数据支撑(Wootton \& Emmerson, 2005)。在营养生 态位维度, 分子生物学和宏条形码技术的进一步发 展和应用, 则有可能为物种间营养生态位作用强度 的量化评估提供基础, 具有较好的应用前景。总体 而言, 由于食肉动物的稀少性和活动隐蔽性, 研究 人员多采用监测性研究而难以开展控制性实验, 未 来需要在食肉动物种间作用强度评估中进行理论 体系和方法技术上的创新(Sévêque et al, 2020)。

(6)深入探究气候变化对食肉动物交互作用与 共存的影响。大型食肉动物大多具有独居、繁殖率 低、种间竞争激烈的特点, 容易最先受到气候变化 的影响。猞猁(Lynx lynx)、貂熊(Gulo gulo)、棕熊 (Ursus arctos)、北极熊(U. maritimus)等高纬度分布
物种和雪豹等高海拔分布的物种, 其栖息地受到气 候变化影响的风险更高, 此类物种应受到优先关注 (Scully et al, 2018)。未来的研究方向可尝试使用气 候因子对物种关系和种群变化进行解释(Yan et al, 2013), 同时结合对未来气候情境的预测, 关注气候 变化是怎样沿食物链以“上行效应”模式对食肉动物 共存产生影响。

(7)在红外相机技术应用中进行技术创新与系 统整合。红外相机技术已经在食肉动物及其猎物的 研究与保护中得到广泛利用(O’Connell et al, 2010; Steenweg et al, 2017), 但近年来红外相机自动监测 技术研发进展缓慢。红外相机长期处于记录声音和 影像阶段, 而在个体标记、声景记录监测、动物体 重和移动速度评估、微距和三维立体拍摄等领域少 有突破, 制约了野生动物区域共存研究的发展。将 模块化技术应用于红外相机监测可有效增加红外 相机应对不同研究背景的灵活性, 同时对经济性也 有较好兼顾。自然资源管理物联网系统可对红外相 机、定位追踪项圈、采样调查和其他传感器信息进 行有效整合, 结合云计算、大数据分析、机器视觉 人工智能和地理信息等技术, 使食肉动物监测研究 走向系统化、规范化、体系化, 成为未来食肉动物 研究与保护的必然趋势(Norouzzadeh et al, 2018; Tabak et al, 2019)。

致谢: 衷心感谢两位匿名审稿专家及编委对本文 提出的宝贵意见和建议。

\section{ORCID}

李治霖 (1D https://orcid.org/0000-0001-6384-601X

李戬 (D) https://orcid.org/0000-0001-7200-9539

王天明 (D) https://orcid.org/0000-0003-3370-0209

\section{参考文献}

Ait Kaci Azzou S, Aebischer T, Singer L, Wolf B, Wegmann D (2019) A sparse occupancy model to quantify species interactions in time and space. BioRxiv, 815027.

Allen ML, Peterson B, Krofel M (2018) No respect for apex carnivores: Distribution and activity patterns of honey badgers in the Serengeti. Mammalian Biology, 89, 90-94.

Andersen GE, Johnson CN, Jones ME (2020) Space use and temporal partitioning of sympatric Tasmanian devils and spotted-tailed quolls. Austral Ecology, 45, 355-365.

Andheria AP, Karanth KU, Kumar NS (2007) Diet and prey profiles of three sympatric large carnivores in Bandipur 
Tiger Reserve, India. Journal of Zoology, 273, 169-175.

Balme GA, Miller JRB, Pitman RT, Hunter LTB (2017) Caching reduces kleptoparasitism in a solitary, large felid. Journal of Animal Ecology, 86, 634-644.

Bhattarai BP, Kindlmann P (2012) Interactions between Bengal tiger (Panthera tigris) and leopard (Panthera pardus): Implications for their conservation. Biodiversity and Conservation, 21, 2075-2094.

Bischof R, Ali H, Kabir M, Hameed S, Nawaz MA (2014) Being the underdog: An elusive small carnivore uses space with prey and time without enemies. Journal of Zoology, 293, 40-48.

Bohmann K, Evans A, Gilbert MTP, Carvalho GR, Creer S, Knapp M, Yu DW, de Bruyn M (2014) Environmental DNA for wildlife biology and biodiversity monitoring. Trends in Ecology \& Evolution, 29, 358-367.

Brashares JS, Prugh LR, Stoner CJ, Epps CW (2010) Ecological and conservation implications of mesopredator release. In: Trophic Cascades: Predators, Prey, and the Changing Dynamics of Nature (ed. Zdilla KM), pp. 221-240. Island Press, Washington, DC.

Broekhuis F, Grunewalder S, McNutt JW, MacDonald DW (2014) Optimal hunting conditions drive circalunar behavior of a diurnal carnivore. Behavioral Ecology, 25, 1268-1275.

Brook LA, Johnson CN, Ritchie EG (2012) Effects of predator control on behaviour of an apex predator and indirect consequences for mesopredator suppression. Journal of Applied Ecology, 49, 1278-1286.

Bruno JF, Stachowicz JJ, Bertness MD (2003) Inclusion of facilitation into ecological theory. Trends in Ecology \& Evolution, 18, 119-125.

Bu HL, Wang F, McShea WJ, Lü Z, Wang DJ, Li S (2016) Spatial co-occurrence and activity patterns of mesocarnivores in the temperate forests of Southwest China. PLoS ONE, 11, e0164271.

Carothers JH, Jaksić FM (1984) Time as a niche difference: The role of interference competition. Oikos, 42, 403-406.

Carroll C (2007) Interacting effects of climate change, landscape conversion, and harvest on carnivore populations at the range margin: Marten and lynx in the northern Appalachians. Conservation Biology, 21, 1092-1104.

Carter NH, Linnell JDC (2016) Co-adaptation is key to coexisting with large carnivores. Trends in Ecology \& Evolution, 31, 575-578.

Carter N, Jasny M, Gurung B, Liu J (2015) Impacts of people and tigers on leopard spatiotemporal activity patterns in a global biodiversity hotspot. Global Ecology and Conservation, 3, 149-162.

Choi MB, Woo D, Choi TY (2015) Composition of the insect diet in feces of yellow-throated marten, Martes flavigula, in Jirisan National Park, South Korea. Journal of Ecology and Environment, 38, 389-395.
Chutipong W, Steinmetz R, Savini T, Gale GA (2017) Assessing resource and predator effects on habitat use of tropical small carnivores. Mammal Research, 62, 21-36.

Crooks KR, Burdett ChL, Theobald DM, King SRB, di Marco M, Rondinini C, Boitani L (2017) Quantification of habitat fragmentation reveals extinction risk in terrestrial mammals. Proceedings of the National Academy of Sciences, USA, 114, 7635-7640.

Cusack JJ, Dickman AJ, Kalyahe M, Rowcliffe JM, Carbone C, MacDonald DW, Coulson T (2017) Revealing kleptoparasitic and predatory tendencies in an African mammal community using camera traps: A comparison of spatiotemporal approaches. Oikos, 126, 812-822.

Davis CL, Rich LN, Farris ZJ, Kelly MJ, Di Bitetti MS, Blanco YD, Albanesi S, Farhadinia MS, Gholikhani N, Hamel S, Harmsen BJ, Wultsch C, Kane MD, Martins Q, Murphy AJ, Steenweg R, Sunarto S, Taktehrani A, Thapa K, Tucker JM, Whittington J, Widodo FA, Yoccoz NG, Miller DAW (2018) Ecological correlates of the spatial co-occurrence of sympatric mammalian carnivores worldwide. Ecology Letters, 21, 1401-1412.

de Satgé J, Teichman K, Cristescu B (2017) Competition and coexistence in a small carnivore guild. Oecologia, 184, 873-884.

Di Bitetti MS, de Angelo CD, Di Blanco YE, Paviolo A (2010) Niche partitioning and species coexistence in a Neotropical felid assemblage. Acta Oecologica, 36, 403-412.

Ditchkoff SS, Saalfeld ST, Gibson CJ (2006) Animal behavior in urban ecosystems: Modifications due to human-induced stress. Urban Ecosystems, 9, 5-12.

Donadio E, Buskirk SW (2006) Diet, morphology, and interspecific killing in Carnivora. The American Naturalist, 167, 524-536.

Elmeros M, Mikkelsen DMG, Nørgaard LS, Pertoldi C, Jensen TH, Chriél M (2018) The diet of feral raccoon dog (Nyctereutes procyonoides) and native badger (Meles meles) and red fox (Vulpes vulpes) in Denmark. Mammal Research, 63, 405-413.

Farhadinia MS, Heit DR, Montgomery RA, Johnson PJ, Hobeali K, Hunter LTB, MacDonald DW (2019) Vertical relief facilitates spatial segregation of a high density large carnivore population. Oikos, 129, 346-355.

Farrington JD, Tsering D (2019) Human-snow leopard conflict in the Chang Tang region of Tibet, China. Biological Conservation, 237, 504-513.

Farris ZJ, Gerber BD, Karpanty S, Murphy A, Wampole E, Ratelolahy F, Kelly MJ (2020) Exploring and interpreting spatiotemporal interactions between native and invasive carnivores across a gradient of rainforest degradation. Biological Invasions, 22, 2033-2047.

Farris ZJ, Kelly MJ, Karpanty S, Ratelolahy F (2016) Patterns of spatial co-occurrence among native and exotic carnivores in north-eastern Madagascar. Animal Conservation, 19, 
189-198.

Fedriani JM, Palomares F, Delibes M (1999) Niche relations among three sympatric Mediterranean carnivores. Oecologia, 121, 138-148.

Filazzola A, Brown C, Dettlaff MA, Batbaatar A, Grenke J, Bao T, Peetoom Heida I, Cahill JF Jr (2020) The effects of livestock grazing on biodiversity are multi-trophic: A meta-analysis. Ecology Letters, 23, 1298-1309.

Finke DL, Denno RF (2006) Spatial refuge from intraguild predation: Implications for prey suppression and trophic cascades. Oecologia, 149, 265-275.

Frey S, Fisher JT, Burton AC, Volpe JP (2017) Investigating animal activity patterns and temporal niche partitioning using camera-trap data: Challenges and opportunities. Remote Sensing in Ecology and Conservation, 3, 123-132.

Gómez-Ortiz Y, Monroy-Vilchis O, Castro-Arellano I (2019) Temporal coexistence in a carnivore assemblage from central Mexico: Temporal-domain dependence. Mammal Research, 64, 333-342.

Gómez-Ortiz Y, Monroy-Vilchis O, Mendoza-Martínez GD (2015) Feeding interactions in an assemblage of terrestrial carnivores in central Mexico. Zoological Studies, 54, 16.

Gallo T, Fidino M, Lehrer EW, Magle SB (2017) Mammal diversity and metacommunity dynamics in urban green spaces: Implications for urban wildlife conservation. Ecological Applications, 27, 2330-2341.

Gaynor KM, Hojnowski CE, Carter NH, Brashares JS (2018) The influence of human disturbance on wildlife nocturnality. Science, 360, 1232-1235.

Geldmann J, Joppa LN, Burgess ND (2014) Mapping change in human pressure globally on land and within protected areas. Conservation Biology, 28, 1604-1616.

Gerber BD, Karpanty SM, Randrianantenaina J (2012) Activity patterns of carnivores in the rain forests of Madagascar: Implications for species coexistence. Journal of Mammalogy, 93, 667-676.

Ghoshal A (2011) Impact of Urbanization on Winter Resource Use and Relative Abundance of a Commensal Carnivore, the Red Fox (Vulpes vulpes). $\mathrm{PhD}$ dissertation, Forest Research Institute University, Dehradun.

Gittleman JL (1985) Carnivore body size: Ecological and taxonomic correlates. Oecologia, 67, 540-554.

Gosselink TE, Van Deelen TR, Warner RE, Joselyn MG (2003) Temporal habitat partitioning and spatial use of coyotes and red foxes in east-central Illinois. The Journal of Wildlife Management, 67, 90-103.

Grassel SM, Rachlow JL, Williams CJ (2015) Spatial interactions between sympatric carnivores: Asymmetric avoidance of an intraguild predator. Ecology and Evolution, 5, 2762-2773.

Grinnell J (1917) The niche-relationships of the California thrasher. Auk, 34, 427-433.

Halle S (2000) Ecological relevance of daily activity patterns.
In: Activity Patterns in Small Mammals (eds. Halle S, Stenseth NC), pp. 67-90. Springer, Berlin.

Harihar A, Pandav B, Goyal SP (2011) Responses of leopard Panthera pardus to the recovery of a tiger Panthera tigris population. Journal of Applied Ecology, 48, 806-814.

Haswell PM, Jones KA, Kusak J, Hayward MW (2018) Fear, foraging and olfaction: How mesopredators avoid costly interactions with apex predators. Oecologia, 187, 573-583.

Hayward MW, Slotow R (2009) Temporal partitioning of activity in large African carnivores: Tests of multiple hypotheses. South African Journal of Wildlife Research, 39, 109-125.

Holt RD, Polis GA (1997) A theoretical framework for intraguild predation. The American Naturalist, 149, 745-764.

Hua Y, Vitekere K, Wang J, Zhu MY, Zaman M, Jiang GS (2020) Coexistence of sympatric carnivores in a relatively homogenous landscape and the effects of environmental factors on site occupation. Annales Zoologici Fennici, 57, 47-58.

Hunter L (2020) Field Guide to Carnivores of the World. Bloomsbury Publishing, London.

Hutchinson GE (1959) Homage to Santa Rosalia or why are there so many kinds of animals? The American Naturalist, 93, 145-159.

Hutchinson GE (1957) Concluding remarks Cold Spring Harbor Symposia on Quantitative Biology. Bulletin of Mathematical Biology, 53, 193-213.

Jiang ZG, Liu SY, Wu Y, Jiang XL, Zhou KY (2017) China's mammal diversity (2nd edition). Biodiversity Science, 25, 886-895. (in Chinese with English abstract) [蒋志刚, 刘少 英, 吴毅, 蒋学龙, 周开亚 (2017) 中国哺乳动物多样性 (第2版). 生物多样性, 25, 886-895.]

Karanth KU, Srivathsa A, Vasudev D, Puri M, Parameshwaran R, Kumar NS (2017) Spatio-temporal interactions facilitate large carnivore sympatry across a resource gradient. Proceedings of the Royal Society B: Biological Sciences, 284, 20161860.

Karanth KU, Sunquist ME (1995) Prey selection by tiger, leopard and dhole in tropical forests. Journal of Animal Ecology, 64, 439-450.

Karanth KU, Sunquist ME (2000) Behavioural correlates of predation by tiger (Panthera tigris), leopard (Panthera pardus) and dhole (Cuon alpinus) in Nagarahole, India. Journal of Zoology, 250, 255-265.

Kauhala K, Laukkanen P, von Rége I (1998) Summer food composition and food niche overlap of the raccoon dog, red fox and badger in Finland. Ecography, 21, 457-463.

Kerley LL, Mukhacheva AS, Matyukhina DS, Salmanova E, Salkina GP, Miquelle DG (2015) A comparison of food habits and prey preference of Amur tiger (Panthera tigris altaica) at three sites in the Russian Far East. Integrative Zoology, 10, 354-364. 
Kitchen AM, Gese EM, Schauster ER (2000) Changes in coyote activity patterns due to reduced exposure to human persecution. Canadian Journal of Zoology, 78, 853-857.

Kronfeld-Schor N, Dayan T (2003) Partitioning of time as an ecological resource. Annual Review of Ecology, Evolution, and Systematics, 34, 153-181.

Lanszki J, Heltai M, Kövér G, Zalewski A (2019) Non-linear relationship between body size of terrestrial carnivores and their trophic niche breadth and overlap. Basic and Applied Ecology, 38, 36-46.

Lashley MA, Cove MV, Chitwood MC, Penido G, Gardner B, DePerno CS, Moorman CE (2018) Estimating wildlife activity curves: Comparison of methods and sample size. Scientific Reports, 8, 4173.

Li S (2020) Development progress and outlook of the wildlife camera-trapping networks in China. Biodiversity Science, 28, 1045-1048. (in Chinese) [李晟 (2020) 中国野生动物 红外相机监测网络建设进展与展望. 生物多样性, 28, 1045-1048.]

Li S, William JM, Wang DJ, Gu XD, Zhang XF, Zhang L, Shen XL (2020) Retreat of large carnivores across the giant panda distribution range. Nature Ecology \& Evolution, 4, 1327-1331.

Li S, Wang DJ, Xiao ZS, Li XH, Wang TM, Feng LM, Wang $Y$ (2014) Camera-trapping in wildlife research and conservation in China: Review and outlook. Biodiversity Science, 22, 685-695. (in Chinese with English abstract) [李 晟, 王大军, 肖治术, 李欣海, 王天明, 冯利民, 王云 (2014) 红外相机技术在我国野生动物研究与保护中的应 用与前景. 生物多样性, 22, 685-695.]

Li ZL, Kang AL, Gu JY, Xue YG, Ren Y, Zhu ZW, Liu PQ, Ma JZ, Jiang GS (2017) Effects of human disturbance on vegetation, prey and Amur tigers in Hunchun Nature Reserve, China. Ecological Modelling, 353, 28-36.

Li ZL, Wang TM, Smith JLD, Feng RN, Feng LM, Mou P, Ge JP (2019) Coexistence of two sympatric flagship carnivores in the human-dominated forest landscapes of Northeast Asia. Landscape Ecology, 34, 291-305.

Li ZL, Kang AL, Lang JM, Xue YG, Ren Y, Zhu ZW, Ma JZ, Liu PQ, Jiang GS (2014) On the assessment of big cats and their prey populations based on camera trap data. Biodiversity Science, 22, 725-732. (in Chinese with English abstract) [李治霖, 康䨩黎, 郎建民, 薛延刚, 任毅, 朱志 文, 马建章, 刘培琦, 姜广顺 (2014) 探讨基于红外相机 技术对大型猫科动物及其猎物的种群评估方法. 生物多 样性, 22, 725-732.]

Liu SY, Wu Y (2019) Handbook of the Mammals of China. The Straits Publishing \& Distributing Group, Fuzhou. (in Chinese) [刘少英, 吴毅 (2019) 中国兽类图鉴. 海峡书 局, 福州.]

Lonsinger RC, Gese EM, Waits LP (2015) Evaluating the reliability of field identification and morphometric classifications for carnivore scats confirmed with genetic analysis. Wildlife Society Bulletin, 39, 593-602.

López-Bao JV, Mattisson J, Persson J, Aronsson M, Andrén H (2016) Tracking neighbours promotes the coexistence of large carnivores. Scientific Reports, 6, 23198.

Lovari S, Ventimiglia M, Minder I (2013) Food habits of two leopard species, competition, climate change and upper treeline: A way to the decrease of an endangered species? Ethology Ecology \& Evolution, 25, 305-318.

Lovari S, Pokheral CP, Jnawali SR, Fusani L, Ferretti F (2015) Coexistence of the tiger and the common leopard in a prey-rich area: The role of prey partitioning. Journal of Zoology, 295, 122-131.

Marinho PH, Fonseca CR, Sarmento P, Fonseca C, Venticinque EM (2020) Temporal niche overlap among mesocarnivores in a Caatinga dry forest. European Journal of Wildlife Research, 66, 1-13.

McGee BK, Ballard WB, Nicholson KL, Cypher BL, Lemons PR, Kamler JF (2006) Effects of artificial escape dens on swift fox populations in northwest Texas. Wildlife Society Bulletin, 34, 821-827.

Mella-Méndez I, Flores-Peredo R, Bolívar-Cimé B, Vázquez-Domínguez G (2019a) Effect of free-ranging dogs and cats on medium-sized wild mammal assemblages in urban protected areas of a Mexican city. Wildlife Research, 46, 669-678.

Mella-Méndez I, Flores-Peredo R, Pérez-Torres J, Hernández-González S, González-Uribe DU, Socorro Bolívar-Cimé B (2019b) Activity patterns and temporal niche partitioning of dogs and medium-sized wild mammals in urban parks of Xalapa, Mexico. Urban Ecosystems, 22, 1061-1070.

Miller JRB, Schmitz OJ (2019) Landscape of fear and human-predator coexistence: Applying spatial predator-prey interaction theory to understand and reduce carnivore-livestock conflict. Biological Conservation, 236, 464-473.

Mondal K, Gupta S, Bhattacharjee S, Qureshi Q, Sankar K (2012) Response of leopards to re-introduced tigers in Sariska Tiger Reserve, Western India. International Journal of Biodiversity and Conservation, 4, 228-236.

Monterroso P, Alves PC, Ferreras P (2014) Plasticity in circadian activity patterns of mesocarnivores in Southwestern Europe: Implications for species coexistence. Behavioral Ecology and Sociobiology, 68, 1403-1417.

Monterroso P, Godinho R, Oliveira T, Ferreras P, Kelly MJ, Morin DJ, Waits LP, Alves PC, Mills LS (2019) Feeding ecological knowledge: The underutilised power of faecal DNA approaches for carnivore diet analysis. Mammal Review, 49, 97-112.

Mueller MA, Drake D, Allen ML (2018) Coexistence of coyotes (Canis latrans) and red foxes (Vulpes vulpes) in an urban landscape. PLoS ONE, 13, e0190971.

Mumma MA, Adams JR, Zieminski C, Fuller TK, Mahoney 
SP, Waits LP (2016) A comparison of morphological and molecular diet analyses of predator scats. Journal of Mammalogy, 97, 112-120.

Ngoprasert D, Lynam AJ, Gale GA (2017) Effects of temporary closure of a national park on leopard movement and behaviour in tropical Asia. Mammalian Biology, 82, 65-73.

Nickel BA, Suraci JP, Allen ML, Wilmers CC (2020) Human presence and human footprint have non-equivalent effects on wildlife spatiotemporal habitat use. Biological Conservation, 241, 108383.

Nishimura K (2010) Kleptoparasitism and cannibalism. In: Encyclopedia of Animal Behavior (eds Breed MD, Moore J), pp. 667-675. Academic Press, Oxford.

Norouzzadeh MS, Nguyen A, Kosmala M, Swanson A, Palmer MS, Packer C, Clune J (2018) Automatically identifying, counting, and describing wild animals in camera-trap images with deep learning. Proceedings of the National Academy of Sciences, USA, 115, E5716-E5725.

O’Connell AF, Nichols JD, Karanth KU (2010) Camera Traps in Animal Ecology: Methods and Analyses. Springer Science \& Business Media, Berlin.

O’Malley C, Elbroch LM, Lendrum PE, Quigley H (2018) Motion-triggered video cameras reveal spatial and temporal patterns of red fox foraging on carrion provided by mountain lions. PeerJ, 6, e5324.

Odden M, Wegge P, Fredriksen T (2010) Do tigers displace leopards? If so, why? Ecological Research, 25, 875-881.

Oriol-Cotterill A, Valeix M, Frank LG, Riginos C, Macdonald DW (2015) Landscapes of coexistence for terrestrial carnivores: The ecological consequences of being downgraded from ultimate to penultimate predator by humans. Oikos, 124, 1263-1273.

Palomares F, Caro TM (1999) Interspecific killing among mammalian carnivores. The American Naturalist, 153, 492-508.

Palomares F, Ferreras P, Fedriani JM, Delibes M (1996) Spatial relationships between Iberian lynx and other carnivores in an area of south-western Spain. Journal of Applied Ecology, 33, 5-13.

Parsons AW, Rota CT, Forrester T, Baker-Whatton MC, McShea WJ, Schuttler SG, Millspaugh JJ, Kays R (2019) Urbanization focuses carnivore activity in remaining natural habitats, increasing species interactions. Journal of Applied Ecology, 56, 1894-1904.

Petersen WJ, Savini T, Steinmetz R, Ngoprasert D (2019) Periodic resource scarcity and potential for interspecific competition influences distribution of small carnivores in a seasonally dry tropical forest fragment. Mammalian Biology, 95, 112-122.

Pokheral CP, Wegge P (2019) Coexisting large carnivores: Spatial relationships of tigers and leopards and their prey in a prey-rich area in lowland Nepal. Ecoscience, 26, 1-9.
Polis GA, Holt RD (1992) Intraguild predation: The dynamics of complex trophic interactions. Trends in Ecology \& Evolution, 7, 151-154.

du Preez B, Purdon J, Trethowan P, MacDonald DW, Loveridge AJ (2017) Dietary niche differentiation facilitates coexistence of two large carnivores. Journal of Zoology, 302, 149-156.

Prugh LR, Sivy KJ (2020) Enemies with benefits: Integrating positive and negative interactions among terrestrial carnivores. Ecology Letters, 23, 902-918.

Radloff FGT, Du Toit JT (2004) Large predators and their prey in a southern African savanna: A predator's size determines its prey size range. Journal of Animal Ecology, 73, 410-423.

Ralls K, White PJ (1995) Predation on San Joaquin kit foxes by larger canids. Journal of Mammalogy, 76, 723-729.

Ramakrishnan U, Coss RG, Pelkey NW (1999) Tiger decline caused by the reduction of large ungulate prey: Evidence from a study of leopard diets in southern India. Biological Conservation, 89, 113-120.

Ramesh T, Kalle R, Sankar K, Qureshi Q (2012) Spatio-temporal partitioning among large carnivores in relation to major prey species in Western Ghats. Journal of Zoology, 287, 269-275.

Richmond OMW, Hines JE, Beissinger SR (2010) Two-species occupancy models: A new parameterization applied to co-occurrence of secretive rails. Ecological Applications, 20, 2036-2046.

Ripple WJ, Estes JA, Beschta RL, Wilmers CC, Ritchie EG, Hebblewhite M, Berger J, Elmhagen B, Letnic M, Nelson MP, Schmitz OJ, Smith DW, Wallach AD, Wirsing AJ (2014) Status and ecological effects of the world's largest carnivores. Science, 343, 1241484.

Ritchie EG, Johnson CN (2009) Predator interactions, mesopredator release and biodiversity conservation. Ecology Letters, 12, 982-998.

Roper TJ (1992) Badger Meles meles setts-Architecture, internal environment and function. Mammal Review, 22, 43-53.

Šálek M, Drahníková L, Tkadlec E (2015) Changes in home range sizes and population densities of carnivore species along the natural to urban habitat gradient. Mammal Review, 45, 1-14.

Salo P, Nordstrom M, Thomson RL, Korpimaki E (2008) Risk induced by a native top predator reduces alien mink movements. Journal of Animal Ecology, 77, 1092-1098.

Sæbø JS (2016) Spatial and Temporal Distributions and Interactions in a Neotropical Ground-Dwelling Animal Community. Master dissertation, Norwegian University, Ås.

Santini L, González-Suárez M, Russo D, Gonzalez-Voyer A, von Hardenberg A, Ancillotto L (2019) One strategy does not fit all: Determinants of urban adaptation in mammals. Ecology Letters, 22, 365-376.

Santos F, Carbone C, Wearn OR, Rowcliffe JM, Espinosa S, 
Lima MGM, Ahumada JA, Gonçalves ALS, Trevelin LC, Alvarez-Loayza P, Spironello WR, Jansen PA, Juen L, Peres C (2019) Prey availability and temporal partitioning modulate felid coexistence in Neotropical forests. PLoS ONE, 14, e0213671.

Sévêque A, Gentle LK, López-Bao JV, Yarnell RW, Uzal A (2020) Human disturbance has contrasting effects on niche partitioning within carnivore communities. Biological Reviews, 95, 1689-1705.

Scharf FS, Juanes F, Rountree RA (2000) Predator size-prey size relationships of marine fish predators: Interspecific variation and effects of ontogeny and body size on trophic-niche breadth. Marine Ecology Progress Series, 208, 229-248.

Schieltz JM, Rubenstein DI (2016) Evidence based review: Positive versus negative effects of livestock grazing on wildlife. What do we really know? Environmental Research Letters, 11, 113003.

Schoener TW (1974) Resource partitioning in ecological communities. Science, 185, 27-39.

Schoener TW (1982) The controversy over interspecific competition: Despite spirited criticism, competition continues to occupy a major domain in ecological thought. American Scientist, 70, 586-595.

Scully AE, Fisher S, Miller DAW, Thornton DH (2018) Influence of biotic interactions on the distribution of Canada lynx (Lynx canadensis) at the southern edge of their range. Journal of Mammalogy, 99, 760-772.

Seidensticker J (1976) On the ecological separation between tigers and leopards. Biotropica, 8, 225-234.

Shamoon H, Saltz D, Dayan T (2017) Fine-scale temporal and spatial population fluctuations of medium sized carnivores in a Mediterranean agricultural matrix. Landscape Ecology, 32, 1243-1256.

Shao XN, Song DZ, Huang QW, Li S, Yao M (2019) Fast surveys and molecular diet analysis of carnivores based on fecal DNA and metabarcoding. Biodiversity Science, 27, 543-556. (in Chinese with English abstract) [邵昕宁, 宋大 昭, 黄巧雯, 李晟, 姚蒙 (2019) 基于粪便DNA及宏条形 码技术的食肉动物快速调查及食性分析. 生物多样性, 27, 543-556.]

Shen XL, Li S, McShea WJ, Wang DJ, Yu JP, Shi XG, Dong W, Mi XC, Ma KP (2020) Effectiveness of management zoning designed for flagship species in protecting sympatric species. Conservation Biology, 34, 158-167.

Silva-Rodríguez EA, Sieving KE (2012) Domestic dogs shape the landscape-scale distribution of a threatened forest ungulate. Biological Conservation, 150, 103-110.

Simcharoen A, Simcharoen S, Duangchantrasiri S, Bump J, Smith JLD (2018) Tiger and leopard diets in western Thailand: Evidence for overlap and potential consequences. Food Webs, 15, e00085.

Soh YH, Carrasco LR, Miquelle DG, Jiang JS, Yang J, Stokes
EJ, Tang JR, Kang AL, Liu PQ, Rao M (2014) Spatial correlates of livestock depredation by Amur tigers in Hunchun, China: Relevance of prey density and implications for protected area management. Biological Conservation, 169, 117-127.

Spencer K, Sambrook M, Bremner-Harrison S, Cilliers D, Yarnell RW, Brummer R, Whitehouse-Tedd K (2020) Livestock guarding dogs enable human-carnivore coexistence: First evidence of equivalent carnivore occupancy on guarded and unguarded farms. Biological Conservation, 241, 108256.

St-Pierre C, Ouellet JP, Crête M (2006) Do competitive intraguild interactions affect space and habitat use by small carnivores in a forested landscape? Ecography, 29, 487-496.

Steenweg R, Hebblewhite M, Kays R, Ahumada J, Fisher JT, Burton C, Townsend SE, Carbone C, Rowcliffe JM, Whittington J, Brodie J, Royle JA, Switalski A, Clevenger AP, Heim N, Rich LN (2017) Scaling-up camera traps: Monitoring the planet's biodiversity with networks of remote sensors. Frontiers in Ecology and the Environment, $15,26-34$.

Steinmetz R, Seuaturien N, Chutipong W (2013) Tigers, leopards, and dholes in a half-empty forest: Assessing species interactions in a guild of threatened carnivores. Biological Conservation, 163, 68-78.

Steinmetz R, Seuaturien $\mathrm{N}$, Intanajitjuy $\mathrm{P}$, Inrueang $\mathrm{P}$, Prempree K (2020) The effects of prey depletion on dietary niches of sympatric apex predators in Southeast Asia. Integrative Zoology, 16, 19-32.

Støen OG, Ordiz A, Evans AL, Laske TG, Kindberg J, Frobert O, Swenson JE, Arnemo JM (2015) Physiological evidence for a human-induced landscape of fear in brown bears (Ursus arctos). Physiology \& Behavior, 152, 244-248.

Sunarto S, Kelly MJ, Parakkasi K, Hutajulu MB (2015) Cat coexistence in central Sumatra: Ecological characteristics, spatial and temporal overlap, and implications for management. Journal of Zoology, 296, 104-115.

Tabak MA, Norouzzadeh MS, Wolfson DW, Sweeney SJ, VerCauteren KC, Snow NP, Halseth JM, Di Salvo PA, Lewis JS, White MD, Teton B, Beasley JC, Schlichting PE, Boughton RK, Wight B, Newkirk ES, Ivan JS, Odell EA, Brook RK, Lukacs PM, Moeller AK, Mandeville EG, Clune J, Miller RS (2019) Machine learning to classify animal species in camera trap images: Applications in ecology. Methods in Ecology and Evolution, 10, 585-590.

Tobler MW, Kéry M, Hui FKC, Guillera-Arroita G, Knaus P, Sattler T (2019) Joint species distribution models with species correlations and imperfect detection. Ecology, 100, e02754.

Torretta E, Serafini M, Puopolo F, Schenone L (2016) Spatial and temporal adjustments allowing the coexistence among carnivores in Liguria (N-W Italy). Acta Ethologica, 19, 123-132. 
Vanak AT, Fortin D, Thaker M, Ogden M, Owen C, Greatwood S, Slotow R (2013) Moving to stay in place: Behavioral mechanisms for coexistence of African large carnivores. Ecology, 94, 2619-2631.

Vanak AT, Gompper ME (2009) Dogs Canis familiaris as carnivores: Their role and function in intraguild competition. Mammal Review, 39, 265-283.

Vilella M, Ferrandiz-Rovira M, Sayol F (2020) Coexistence of predators in time: Effects of season and prey availability on species activity within a Mediterranean carnivore guild. Ecology and Evolution, 10,'11422-11408.

Wallach AD, Izhaki I, Toms JD, Ripple WJ, Shanas U (2015) What is an apex predator? Oikos, 124, 1453-1461.

Wang F, McShea WJ, Li S, Wang DJ (2018) Does one size fit all? A multispecies approach to regional landscape corridor planning. Diversity and Distributions, 24, 415-425.

Wang SW, Macdonald DW (2009) Feeding habits and niche partitioning in a predator guild composed of tigers, leopards and dholes in a temperate ecosystem in central Bhutan. Journal of Zoology, 277, 275-283.

Wang TM, Feng LM, Mou P, Wu JG, Smith JLD, Xiao WH, Yang HT, Dou HL, Zhao XD, Cheng YC, Zhou B, Wu HY, Zhang L, Tian Y, Guo QX, Kou XJ, Han XM, Miquelle DG, Oliver CD, Xu RM, Ge JP (2016) Amur tigers and leopards returning to China: Direct evidence and a landscape conservation plan. Landscape Ecology, 31, 491-503.

Wang TM, Feng LM, Yang HT, Han BY, Zhao YH, Juan L, Lü X, Zou L, Li T, Xiao WH, Mou P, Smith JLD, Ge JP (2017) A science-based approach to guide Amur leopard recovery in China. Biological Conservation, 210, 47-55.

Wang YW, Allen ML, Wilmers CC (2015) Mesopredator spatial and temporal responses to large predators and human development in the Santa Cruz Mountains of California.
Biological Conservation, 190, 23-33.

Wilmers CC, Wang YW, Nickel B, Houghtaling P, Shakeri Y, Allen ML, Kermish-Wells J, Yovovich V, Williams T (2013) Scale dependent behavioral responses to human development by a large predator, the puma. PLoS ONE, 8, e60590.

Wootton JT, Emmerson M (2005) Measurement of interaction strength in nature. Annual Review of Ecology, Evolution \& Systematics, 36, 419-444.

Yan CC, Stenseth NC, Krebs CJ, Zhang ZB (2013) Linking climate change to population cycles of hares and lynx. Global Change Biology, 19, 3263-3271.

Yen SC, Ju YT, Shaner PL, Chen HL (2019) Spatial and temporal relationship between native mammals and free-roaming dogs in a protected area surrounded by a metropolis. Scientific Reports, 9, 8161.

Zapata- Ríos G, Branch LC (2016) Altered activity patterns and reduced abundance of native mammals in sites with feral dogs in the high Andes. Biological Conservation, 193, 9-16.

Zhao GJ, Yang HT, Xie B, Gong YN, Ge JP, Feng LM (2020) Spatio-temporal coexistence of sympatric mesocarnivores with a single apex carnivore in a fine-scale landscape. Global Ecology and Conservation, 21, e00897.

Zhang LJ, Wang AM, Yuan L, Bao WD, Yang YX, Ba TE (2011) Preliminary comparison of diet composition of four small sized carnivores at Saihanwula Nature Reserve, Inner Mongolia. Acta Theriologica Sinica, 31, 55-61. (in Chinese with English abstract) [张雨嘉, 王安梦, 袁梨, 鲍伟东, 杨 永所，巴特尔 (2011) 内蒙古赛罕乌拉自然保护区4种小 型食肉目动物的食性构成的初步分析. 兽类学报, 31 , 55-61.]

(责任编委: 李涁彬 责任编辑: 周玉荣) 